\title{
Article
}

\section{Is There a Fundamental Upper Limit to the Mass of a Star Cluster?}

Norris, Mark A., van de Ven, Glenn, Kannappan, Sheila J., Schinnerer, Eva and Leaman, Ryan

Available at http://clok.uclan.ac.uk/29392/

Norris, Mark A. ORCID: 0000-0002-7001-805X, van de Ven, Glenn, Kannappan, Sheila J., Schinnerer, Eva and Leaman, Ryan (2019) Is There a Fundamental Upper Limit to the Mass of a Star Cluster? Monthly Notices Of The Royal Astronomical Society, 488 (4). pp. 5400-5408. ISSN 0035-8711

It is advisable to refer to the publisher's version if you intend to cite from the work. http://dx.doi.org/10.1093/mnras/stz2096

For more information about UCLan's research in this area go to http://www.uclan.ac.uk/researchgroups/ and search for <name of research Group>.

For information about Research generally at UCLan please go to http://www.uclan.ac.uk/research/

All outputs in CLoK are protected by Intellectual Property Rights law, including Copyright law. Copyright, IPR and Moral Rights for the works on this site are retained by the individual authors and/or other copyright owners. Terms and conditions for use of this material are defined in the policies page.

\section{CLoK}

Central Lancashire online Knowledge www.clok.uclan.ac.uk

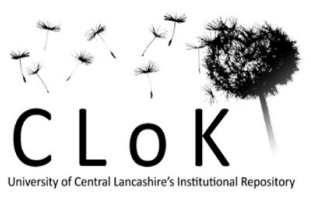




\title{
Is there a fundamental upper limit to the mass of a star cluster?
}

\author{
Mark A. Norris ${ }^{\oplus},{ }^{1,2 \star}$ Glenn van de Ven ${ }^{\oplus},{ }^{2,3,4}$ Sheila J. Kannappan, ${ }^{5}$ Eva Schinnerer ${ }^{2}$ \\ and Ryan Leaman ${ }^{2}$ \\ ${ }^{1}$ Jeremiah Horrocks Institute, University of Central Lancashire, Preston, Lancashire PR1 2HE, UK \\ ${ }^{2}$ Max Planck Institut für Astronomie, Königstuhl 17, D-69117 Heidelberg, Germany \\ ${ }^{3}$ European Southern Observatory, Karl-Schwarzschild-Str. 2, D-85748 Garching bei Munchen, Germany \\ ${ }^{4}$ Department of Astrophysics, University of Vienna, Türkenschanzstrasse 17, A-1180 Vienna, Austria \\ ${ }^{5}$ Department of Physics and Astronomy UNC-Chapel Hill, CB 3255, Phillips Hall, Chapel Hill, NC 27599-3255, USA
}

Accepted 2019 July 26. Received 2019 July 26; in original form 2018 December 8

\begin{abstract}
The discovery around the turn of the millennium of a population of very massive $\left(M_{\star}>2 \times 10^{6}\right.$ $\mathrm{M}_{\odot}$ ) compact stellar systems (CSS) with physical properties (radius, velocity dispersion, stellar mass etc.) that are intermediate between those of the classical globular cluster (GC) population and galaxies led to questions about their exact nature. Recently a consensus has emerged that these objects, usually called ultracompact dwarfs (UCDs), are a mass-dependent mixture of high-mass star clusters and remnant nuclei of tidally disrupted galaxies. The existence of genuine star clusters with stellar masses $>10^{7} \mathrm{M}_{\odot}$ naturally leads to questions about the upper mass limit of the star cluster formation process. In this work we compile a comprehensive catalogue of CSS, and reinforce the evidence that the true ancient star cluster population has a maximum mass of $M_{\star} \sim 5 \times 10^{7} \mathrm{M}_{\odot}$, corresponding to a stellar mass at birth of close to $10^{8} \mathrm{M}_{\odot}$. We then discuss several physical and statistical mechanisms potentially responsible for creating this limiting mass.
\end{abstract}

Key words: galaxies: evolution-galaxies: formation-galaxies: star clusters: general.

\section{INTRODUCTION}

In the last two decades the previously clear distinction between star clusters and galaxies has been blurred by the discovery of new classes of stellar system. Particularly intriguing was the unexpected discovery of a population of luminous, but compact, stellar systems which smoothly extend between the star cluster and galaxy sequences in various observational planes, such as mass-size, effective surface mass density-mass, and velocity dispersion-mass (see e.g. Haşegan et al. 2005; Kissler-Patig, Jordán \& Bastian 2006; Brodie et al. 2011; Misgeld et al. 2011; Norris et al. 2014). These objects, generally called ultracompact dwarfs (UCDs: Minniti et al. 1998; Hilker et al. 1999; Drinkwater et al. 2000; Phillipps et al. 2001) posed a major problem as they were not easily classifiable as either star clusters or galaxies. This led to much discussion over whether these objects were merely the high-mass (and physically extended) tail of the normal globular cluster (GC) population (e.g. Fellhauer \& Kroupa 2002; Mieske, Hilker \& Misgeld 2012), or were in fact the remnant nuclei of dwarf galaxies tidally disrupted through interactions with larger companions (Bekki \& Couch 2003; Pfeffer \& Baumgardt 2013).

Additionally, over the same period a further complication arose that makes it even more difficult to separate bonafide star clusters

^E-mail: mnorris2@uclan.ac.uk from galaxies and hence to determine which formation channel is responsible for creating UCDs. This was the discovery that most Milky Way GCs are not strictly true single stellar populations (SSPs), but in fact display complex abundance spreads (see e.g. Gratton, Carretta \& Bragaglia 2012). This discovery complicated the use of one of the simplest discriminators between GCs and galaxies and raised questions about what the true definition of a star cluster or galaxy should be (Forbes \& Kroupa 2011; Willman $\&$ Strader 2012). For the purposes of this work we define galaxies as those objects located at the bottom of a potential well created by a combination of baryons and dark matter. This location means that they have the potential to acquire additional gas over time and can undergo repeated periods of star formation and metallicity enrichment. Star clusters lack this privileged position and are therefore limited to forming stars using only the gas they are born from, or from any gas they can hold on to as it is released by stellar evolution. Therefore, their stellar populations are necessarily simpler, and their stars cannot, for example, display broad Fe-peak metallicity distributions seen in even the lowest mass Milky Way satellite galaxies (see e.g. Koch et al. 2006; Starkenburg et al. 2013; Hendricks et al. 2014).

Fortunately, based on significantly increased data samples, in recent years a consensus has begun to emerge that both suggested channels are responsible for forming UCDs (Hilker 2006; Brodie et al. 2011; Chiboucas et al. 2011; Norris \& Kannappan 2011; Forbes et al. 2014; Norris et al. 2014; Pfeffer et al. 2014, 2016; 
Voggel, Hilker \& Richtler 2016). This change was motivated by the observation that while the numbers of UCDs are in general in excellent agreement with those expected from an extrapolation of the GC luminosity function (GCLF; Hilker 2006; Norris \& Kannappan 2011; Mieske et al. 2012), an increasing number of cases of definitively stripped nuclei UCDs do exist (Norris \& Kannappan 2011; Seth et al. 2014; Jennings et al. 2015; Norris et al. 2015; Ahn et al. 2017, 2018). Furthermore, cosmological simulations indicate that stripped nuclei could make up a significant fraction of the UCD population only at the highest masses $\left(>10^{7} \mathrm{M}_{\odot}\right)$, and should be a relatively negligible component $(<10$ per cent $)$ at the lowest masses (Pfeffer et al. 2014).

This realization has led to a shifting of emphasis towards finding diagnostics to determine which route was at work for particular objects. It is relatively straightforward to classify some objects as former nuclei; if they are still associated with stellar or gaseous debris streams (Norris \& Kannappan 2011; Jennings et al. 2015; Schweizer et al. 2018), display complex multicomponent structures or even their own associated GC systems (Haşegan et al. 2005; Voggel et al. 2016), contain a supermassive black hole (Seth et al. 2014; Ahn et al. 2017, 2018; Afanasiev et al. 2018), display extreme metallicities only found in the central regions of galaxies (Janz et al. 2016), or exhibit an extended star formation history (Norris et al. 2015; Schweizer et al. 2018). For other objects no definitive signature of their origin might persist. For example, because the object is a hybrid; a true massive star cluster which through dynamical friction sank to the centre of a dwarf galaxy to become its nucleus (one of the proposed origins of such nuclei, see e.g. Georgiev \& Böker 2014), and which was subsequently left behind when the surrounding galaxy was stripped by a tidal interaction (see e.g. Goodman \& Bekki 2018).

As part of this effort, based on extrapolation of the empirically observed GCLF, Norris \& Kannappan (2011) suggested the existence of an upper luminosity/mass limit for true star clusters. Given the properties of the GCLF; its approximately Gaussian shape (see e.g. Jordán et al. 2007; Faifer et al. 2011; Harris et al. 2014), universal turnover magnitude (Strader et al. 2006), and weak trend of increasing GCLF width with galaxy mass (Jordán et al. 2007), it is possible to estimate the luminosity of the brightest GCs expected to be found in a given GC system. This approach provides a remarkably good match to the observed behaviour that the mass of the most massive GC in a GC system correlates strongly with total GC system size (Hilker 2009; Norris \& Kannappan 2011). Norris \& Kannappan (2011) additionally found that given that the richest GC systems have around 10000-20000 members (those found around cD galaxies like M87), the most luminous GC-type UCD should have $M_{V} \sim-13$, which for old stellar systems approximates to $7 \times 10^{7} \mathrm{M}_{\odot}$.

One important caveat to this argument is that the GC systems of galaxies are composite, built-up from GCs formed in situ and those accreted from smaller companion galaxies (see e.g. Forbes \& Bridges 2010; Leaman, VandenBerg \& Mendel 2013). This implies that the total GC population available to produce a most massive GC will be less than that implied by the present GC system size, as the accreted lower mass galaxies will not contribute particularly massive GCs (due to the previously described trend of smaller GC systems having most massive GCs of lower mass). This effect has been observed to have important implications, for example simulations indicate that it leads to the production of the 'blue tilt' observed in GC systems, whereby more massive GCs are on average redder and more metal rich (Choksi, Gnedin \& Li 2018; Usher et al. 2018). This is explained by the fact that lower mass galaxies are lower metallicity and can only produce lower metallicity and lower mass GCs, meaning fewer low-metallicity GCs exist moving up the GC luminosity function, thereby changing the average GC metallicity as a function of GC mass.

Given the fact that many if not most GCs of massive galaxies are accreted, as evidenced by the fact that simulations indicate that as much of 80 per cent of the stellar mass of massive galaxies forms ex situ and is later accreted (Oser et al. 2010; Rodriguez-Gomez et al. 2016; Clauwens et al. 2018; Choksi \& Gnedin 2019), we must reduce the effective GC system size, removing those GCs formed in low-mass galaxies, which cannot produce massive GCs. If the GC system formed in situ around a cD galaxy is reduced to around 10000 members, implying a 50 per cent accreted fraction (in line with typical accreted stellar mass fractions for massive galaxies: Rodriguez-Gomez et al. 2016; Qu et al. 2017), the predicted maximum GC luminosity drops to $M_{V} \sim-12.5$, leading to a mass limit of around $4 \times 10^{7} \mathrm{M}_{\odot}$. This value is consistent with the mass regime where Janz et al. (2016) observe a transition in the metallicity distribution of GCs/UCDs, with objects more massive than a few $\times 10^{7} \mathrm{M}_{\odot}$ exclusively displaying extremely high metallicities. It is also consistent with the $2 \times 10^{7} \mathrm{M}_{\odot}$ limit above which Pfeffer et al. (2016) find that Virgo and Fornax UCDs can be entirely explained by the expected number of stripped nuclei. Hence we propose that there should exist a limiting mass for a genuine old GC of around $4 \times 10^{7} \mathrm{M}_{\odot}$.

With the advent of recent more comprehensive searches for UCDs it is now possible to revisit this prediction. This paper is organized as follows. Section 2 describes the construction of a catalogue of massive compact stellar systems (CSS), Section 3 examines the luminosity function of CSSs for evidence of a truncation of true star clusters. Section 4 provides some suggestions for mechanisms which could be responsible for creating the observed truncation, Section 5 provides a general discussion, and finally Section 6 provides some concluding remarks.

\section{CATALOGUE}

Until recently the principle problem limiting the study of massive CSS was a historic preference in studies of GC systems to enforce either an upper magnitude or a size limit on the selected GC candidates, in order to reduce contamination from background galaxies. The relaxation of these limits (in order to allow UCDs into the selection), along with very deep spectroscopic surveys which are typically complete down to $M_{V} \sim-11$ (e.g. Mieske, Hilker \& Infante 2004; Da Rocha et al. 2011; Misgeld et al. 2011; Mieske et al. 2012), and systematic searches for exactly the type of objects previously excluded (see e.g. Norris et al. 2014) has allowed for the compilation of large catalogs of CSS spanning the GC to galaxy regimes.

While these catalogues are by no means homogeneous, or complete, especially at low luminosities/masses, the fact that the most extended and luminous objects are the easiest to find and spectroscopically confirm ensures that the census of massive UCDs is close to complete for the area surveyed. Therefore, until truly volume-limited spectroscopically confirmed samples selected from surveys such as the Next Generation Virgo Cluster Survey (NGVS; Ferrarese et al. 2012) become available, these compilations remain the most comprehensive.

In this work we compile the most extensive catalogue of spectroscopically confirmed CSSs, in order to search for a truncation in the upper mass of star clusters. The principle sources for the catalogue are the previous compilations of Brodie et al. (2011), Misgeld \& 
Hilker (2011), and in particular the Archive of Intermediate Mass Stellar Systems (AIMSS; Forbes et al. 2014; Norris et al. 2014; Janz et al. 2016). These compilations include the Coma Cluster UCD's of Chiboucas et al. (2011), the Perseus Cluster UCD sample of Penny, Forbes \& Conselice (2012) and Penny et al. (2014), the Antlia Cluster UCD sample of Caso et al. (2013, 2014), the Centaurus A UCDs of Taylor et al. (2010), and the NGC 1132 UCD's of Madrid \& Donzelli (2013).

To these catalogues we add additional M87 UCDs from Haşegan et al. (2005) and Zhang et al. (2015), the UCD of NGC 5044 (Faifer et al. 2017), the UCD of NGC 7727 (Schweizer et al. 2018), GCs from the Milky Way system (Harris 1996, 2010 edition), M31 GCs (Galleti et al. 2004), and GCs of the Hydra I cluster Misgeld et al. (2011). Finally we include the sample of GCs detected in the ACS Virgo Cluster Survey (ACSVCS; Côté et al. 2004; Jordán et al. 2007), this sample is not spectroscopically confirmed, but due to the excellent HST imaging, contamination of the high-confidence GC sample (we select only objects with GC probability $>95$ per cent) is expected to be negligible.

Ideally we would examine the mass of the CSSs directly. However, due to the extreme inhomogeneity of the available photometry this is not possible. We therefore examine the distribution of absolute $V$ magnitudes, as these are most readily available in the literature and are a good proxy for stellar mass for old stellar systems. The only limitation we impose is to exclude the handful of CSSs with spectroscopically derived ages $<3 \mathrm{Gyr}$, so that younger CSSs do not appear artificially bright when compared to the majority older population. This removes only a handful of young clusters from nearby merger systems (such as NGC 7252), plus a few suspected stripped-nucleus type UCDs.

\section{RESULTS}

Fig. 1 shows the location of our CSS sample, plotted in the luminosity-size plane. Other dynamically hot stellar systems are also plotted for illustrative purposes. This plot shows that despite the fact that objects tend to scatter diagonally (due to common dependence of the absolute magnitude and physical effective radius on the distance estimation), approximately along the line connecting GCs and galaxies, very few objects are consistent with being more luminous than $M_{V}<-13$ and more compact than $R_{\mathrm{e}} \sim 200$ pc. Furthermore, the 7 UCDs which are unambiguous stripped nuclei (M60-UCD1, NGC 4546-UCD1, M59cO, VUCD3, UCD3, M59UCD3, NGC 7727-Nucleus 2 indicated by orange circles) are all broadly consistent with being $M_{V}=-13$ or brighter. The remaining objects more luminous than $M_{V}=-13$ either have not yet been studied in detail, or have no definitive evidence to prove their type either way.

We omit an examination of the cE population, which the UCD population may overlap with somewhat, due to their ambiguous origin, and the fact that they are unambiguously galaxies not massive star clusters. While it seems clear that many cEs are the result of tidal stripping interactions (Huxor et al. 2011), there is also the possibility that there may also be a population of intrinsically compact elliptical galaxies, analogous or related to the massive compact galaxies observed at higher redshift (see e.g. Kormendy et al. 2009; van der Wel et al. 2014). Even within the stripping scenario a diverse range of objects may result depending on whether the stripped galaxy is gas rich or already quenched. Going forward, our references to stripping formation scenarios should be interpreted to include the gas-rich dwarf accretion scenario of Du et al. (2019), where the dense metal-rich cE (or potentially UCD) is formed during

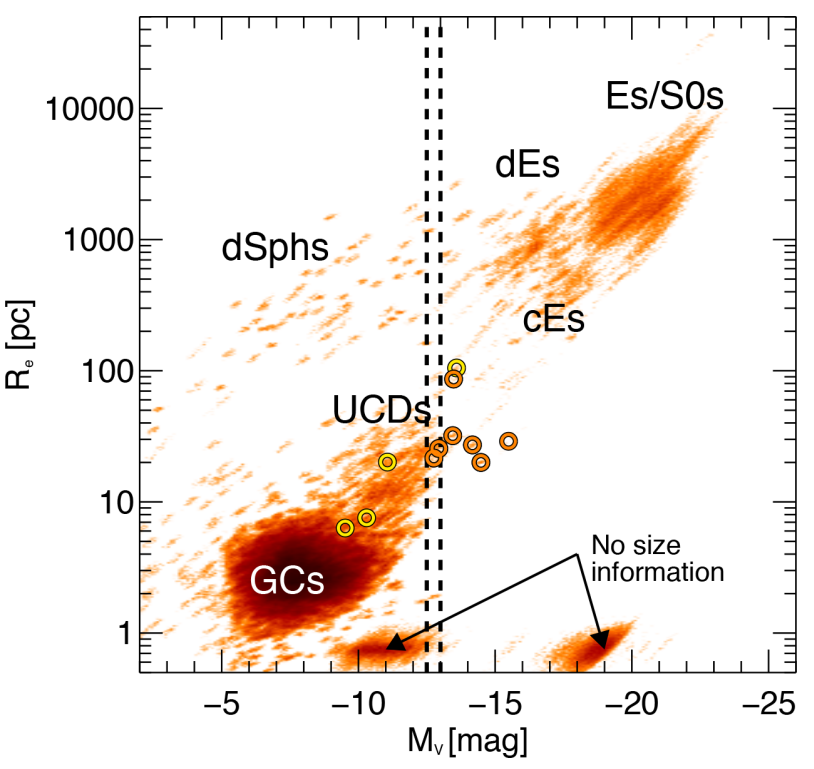

Figure 1. The luminosity-size plane for dynamically hot stellar systems. Rather than plotting points we show the probability density for each object, by including the uncertainties on the distance, size, and magnitude. This more accurately reflects the inherent correlations in the absolute magnitude and radius (caused by their mutual dependence on distance). The clouds of objects with $R_{\mathrm{e}} \sim 0.75 \mathrm{pc}$ and $M_{V} \sim-11$ and -19 are those UCDs and cEs which have no measured size and are therefore given arbitrary size. The six orange open circles indicate those UCDs known to be stripped nuclei (M60-UCD1, NGC 4546-UCD1, M59cO, VUCD3, UCD3, M59UCD3, NGC 7727-Nucleus 2), the yellow open circles are highly suspected stripped nuclei ( $\omega$ Cen, M54, S999, VUCD7). The vertical dashed lines show the proposed region between $M_{V}=-12.5$ and -13 mag where star clusters cease to exist. The dramatic drop-off in numbers of objects in this luminosity range is clear.

the stripping event, by the ram pressure confinement of the gas (and resulting rapid enrichment) of a central starburst triggered by the interaction.

Fig. 2 shows the magnitude distributions of various subsamples of CSSs. The blue histogram is the full catalogue compiled here, and although the constituent surveys all have differing selection criteria, they are all fairly complete for objects brighter than around $M_{V}=-11$ or -11.5 . The red histogram is the distribution of GC luminosities found in imaging of 100 Virgo cluster galaxies by the ACSVCS. It can be confidently assumed that if the regions around each of the non-ACSVCS CSSs were surveyed to the same depth as the ACSVCS, the blue histogram would assume an almost identical shape to that of the ACSVCS sample. The green dot-dashed line is not a fit to the red ACSVCS histogram, but instead shows a Gaussian with mean magnitude and dispersion $\sigma$ chosen to match those found for the GC population of M87 $\left(M_{V}\right.$ $=-7.5$ and 1.3 , respectively) and then arbitrarily normalized to match the ACVCS distribution (see Liu et al. 2015 for a similar examination of the GC/UCD population of M87). The filled orange histogram shows the magnitudes of seven confirmed stripped nucleitype CSSs, the filled yellow histogram shows the four strongly suspected former nuclei, and the dashed vertical black lines delineate the regime where true star clusters are proposed to cease to exist.

The close agreement between the model Gaussian and the ACSVCS histogram demonstrates that the CSS distribution for Virgo is well fit by a single luminosity function where the fit 


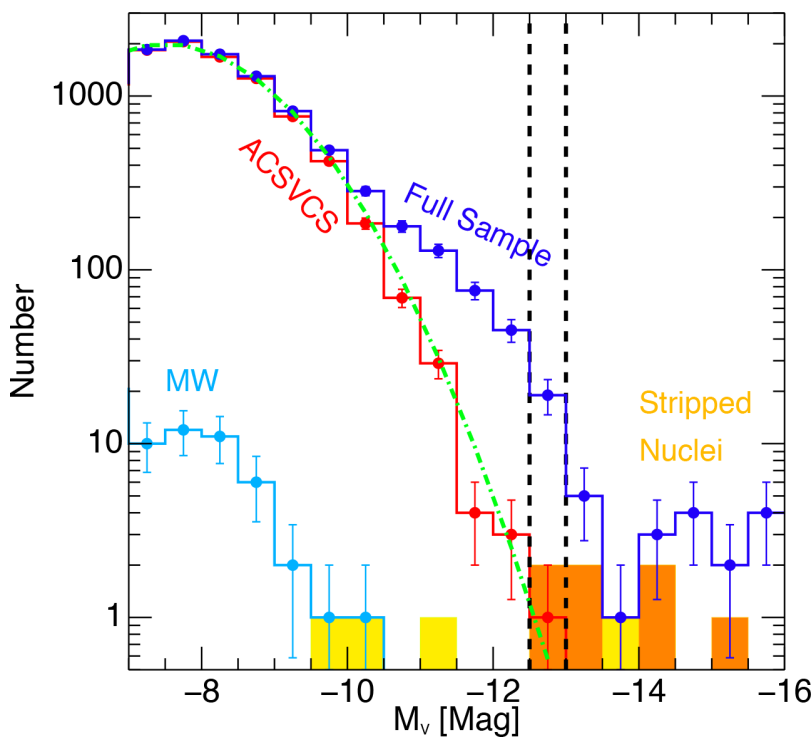

Figure 2. Histogram of $M_{V}$ for samples of CSSs. The blue histogram shows the full catalogue of CSSs. The red histogram shows all objects from the ACSVCS which have $>95$ per cent probability of being GCs or UCDs. The cyan histogram shows the distribution of magnitudes of Milky Way GCs. The filled orange regions show the locations of those CSSs known to be stripped galaxy nuclei, the filled yellow regions indicate suspected stripped galaxy nuclei. The green dot-dashed curve is not a fit to ACSVCS, but shows a Gaussian with central $M_{V}=-7.5 \mathrm{mag}$, dispersion $=1.3 \mathrm{mag}$, and peak of $2000 \mathrm{GCs}$. The vertical dashed lines again show the proposed region between $M_{V}=-12.5$ and -13 mag where true star clusters cease to exist, above $M_{V}=-13$ mag the number of CSSs is consistent with being constant.

parameters are dominated by GCs within a few magnitudes of the turnover magnitude (as is typically the case for estimations of the GCLF of galaxies). The agreement between the upper limit where the green line predicts only a single star cluster, and our suggested upper magnitude limit for star cluster formation is by construction. As discussed in the introduction it was the observation that even GC systems with >10000-20000 members would not predict more than $\sim 1$ GC with magnitude $\lesssim-12.5-13$ that motivated the definition of the upper limit.

The full sample further supports our previous suggestion that $M_{V} \sim-13$ mag marks a transition in the CSS population. There are $19 \mathrm{CSSs}$ in the magnitude bin $-12.5<M_{V}<-13$, but above this the number of objects is approximately constant with only $\sim 3$ per $0.5 \mathrm{mag}$ bin. This levelling-off in the number of objects more luminous than $M_{V}=-13$, despite them being more easily discovered, is evidence for a change in CSS behaviour at this magnitude. To definitely demonstrate the exact value of the transition magnitude will likely require the assembly of true volume-limited and highly complete samples of CSSs, such as those assembled by combining deep imaging surveys such as the NGVS (Ferrarese et al. 2012) with equally complete spectroscopic follow-up.

Our interpretation of this behaviour, that above $M_{V}=-13$ all objects are stripped galaxy substructures, is further supported by the observation that seven of the objects with $M_{V} \lesssim-13$ have already been shown to be ex-nuclei (e.g. Seth et al. 2014; Norris et al. 2015; Ahn et al. 2017). We predict that the majority of objects more luminous than $M_{V}<-13$ will display unambiguous evidence of a galactic origin (some formed by stripping at early epochs may be indistinguishable from star clusters in practice).
From our examination of the stellar masses of CSSs (see e.g. Norris \& Kannappan 2011; Norris et al. 2014; Janz et al. 2016) we found that the suggested $M_{V}=-13 \mathrm{mag}$ limit translates into a current stellar mass of around 3-7 $\times 10^{7} \mathrm{M}_{\odot}$ for these objects. Assuming a Kroupa initial mass function (Kroupa 2001) the stellar mass-loss due to stellar evolution over $10 \mathrm{Gyr}$ is around 30 per cent (Into \& Portinari 2013). Therefore at birth our limit translates to a maximum stellar mass for a true stellar cluster of between $7 \times 10^{7}$ and $10^{8} \mathrm{M}_{\odot}$, depending on the fraction of gas from stellar evolution that the cluster can retain.

A final piece of evidence in favour of the proposed scenario is provided by the observation of young massive star clusters in nearby galaxies. To date, the most massive young star cluster discovered is NGC 7252-W3, which has a mass of $(8 \pm 2) \times 10^{7} \mathrm{M}_{\odot}$ and a radius of $17 \mathrm{pc}$ at an age of around $500 \mathrm{Myr}$ (Maraston et al. 2004). This cluster, along with all other young massive clusters which approach the proposed limit are found associated with ongoing or recent major galaxy mergers, perhaps indicating that unusually violent events are required to create such massive star clusters (see also Bastian et al. 2013), at least at $z=0$.

\section{CAUSES OF THE UPPER MASS LIMIT}

Having demonstrated that the luminosity function of CSSs supports the existence of an upper mass limit for true star clusters of around $10^{8} \mathrm{M}_{\odot}$, we now consider the mechanisms potentially responsible for creating the limit.

\subsection{Scenario A: the need for extreme ISM densities and pressures}

Kruijssen (2012) presents a theoretical scenario in which gravitationally bound star clusters form across the density spectrum of the ISM, but with increasing efficiency at higher densities. This leads naturally to the prediction that to form the most extreme members of the star cluster population requires extreme conditions in the ISM. Unfortunately there have been relatively few simulations that can directly test such a scenario, as the required simulations must capture massive star cluster formation across the full range of ISM conditions found in galaxies or galaxy mergers. In part this lack of suitable simulations is because until recently it has been technically impossible to adequately sample the range of spatial scales involved, as galaxies and mergers typically require examination across tens of kpc, while GCs have half-light radii of only $\sim 2 \mathrm{pc}$.

However, Renaud et al. (2014) and Renaud, Bournaud \& Duc (2015) extending the work of Bournaud, Duc \& Emsellem (2008) presented a hydrodynamical simulation which attempts to reproduce the well-studied ongoing merger system of the Antennae galaxies. This simulation has resolution of $1.5 \mathrm{pc}$ and includes star formation and stellar feedback, allowing a more detailed examination of the properties of young clusters formed in the merger. By comparison with a similar simulation of an isolated Milky Way-like galaxy from Renaud et al. (2013) they are able to contrast the properties of CSS formed in relatively quiescent galaxies, versus those formed in intense merger induced starbursts.

The headline result from the Renaud et al. $(2013,2014,2015)$ simulations are that the MW simulation does not form any star clusters more massive than $3 \times 10^{6} \mathrm{M}_{\odot}$, while the Antennae simulation creates star clusters up to a maximum mass of around $\sim 10^{8} \mathrm{M}_{\odot}$ with radii of $10-30 \mathrm{pc}$, similar to those of UCDs or extreme young massive clusters such as NGC 7252-W3. They find that star clusters up to $5 \times 10^{7} \mathrm{M}_{\odot}$ form in or close to the tidal tails, 
and clusters of up to $10^{8} \mathrm{M}_{\odot}$ form in the densest central regions during the final coalescence (see also Li, Mac Low \& Klessen 2004; Matsui et al. 2012, for simulations that create massive central star clusters of mass $\sim 10^{8} \mathrm{M}_{\odot}$ ). They conclude that the galactic interaction leads to tidally and turbulently compressive regions in the ISM which in turn leads to the formation of clusters 30 times more massive than those found in quiescent discs. One caveat to this work, is that the most massive star clusters formed in the simulations often display an age spread of up to $100 \mathrm{Myr}$, due to ongoing accretion of gas leading to prolonged star formation. Such extended star formation histories are ruled out for modern young massive clusters of mass $10^{6}-10^{7} \mathrm{M}_{\odot}$ (see e.g. Cabrera-Ziri et al. 2014 , 2015), but it is currently not possible to place stringent limits on the length of star formation for any of the bonafide massive UCDs of our sample, due to their distance, and hence unresolved stellar populations.

Nevertheless, more recent studies find broadly similar results to the studies of Renaud et al. (2013, 2014, 2015). For example, when studying the formation of bound stellar clusters in simulated interacting galaxies (Maji et al. 2017) find that clusters as massive as $10^{7.5} \mathrm{M}_{\odot}$ can be formed, but they form preferentially in the most highly shocked regions of galaxy interactions where the pressure is $10^{4}-10^{8}$ times larger than typical for the ISM. Similarly, based on high-resolution simulations Ma et al. (2019) find that bound clusters form preferentially in high-pressure, high-density environments, and further suggest that external pressure (from colliding clouds/gas streams or feedback winds) is required to produce the necessary pressures to form proto-GCs. Other studies have likewise found that merger induced interactions may be required to produce sufficiently high pressures and densities to produce star clusters significantly above the typical turnover mass for GCs of $2 \times 10^{5} \mathrm{M}_{\odot}$ (see e.g. $\mathrm{Li}$ et al. 2017; Kim et al. 2018).

In conclusion it seems that in order to form massive star clusters it is necessary to have very high gas densities with significantly higher compression (due to turbulence) than is present in presentday quiescent discs. However, it is clear that the necessary gas densities and turbulence appear to have been much more common at higher redshift when the bulk of the massive star cluster population was formed, even in the so-called clumpy discs commonly observed at higher $z$ (see e.g. Swinbank et al. 2011; Falgarone et al. 2017). It is also interesting to note that significant samples of objects with masses $\left(10^{6}\right.$ to $\left.2 \times 10^{7} \mathrm{M}_{\odot}\right)$ and sizes expected of the progenitors of modern massive star clusters are beginning to be resolved in studies of lensed galaxies at $z=3-8$, exactly when the bulk of GC progenitors are expected to form (Bouwens et al. 2017; Vanzella et al. 2017).

The observation that very high gas densities and pressures are required to form massive star clusters naturally leads to a limitation on the maximum mass of a cluster that can form (see also Elmegreen 2018 for similar arguments relating to the formation of GCs in highredshift galaxies). Even in such a large merger as the Antennae the physical conditions never reach the threshold required to form clusters of $10^{8} \mathrm{M}_{\odot}$, except in the very central regions of the merger, where any clusters that form are quickly incorporated into the bulge. For star clusters formed in the tidal tails (i.e. those formed on orbits that could allow them to survive for a Hubble time) the maximum compression is necessarily lower than that reached in the central regions at the bottom of the galactic potential. This is because the gas in tidal tails can expand outwards perpendicular to the gas inflow along the tidal tail, whereas in the central regions additional infalling gas can keep the gas pressure high allowing higher mass clusters to form.

\subsection{Scenario B: insufficient molecular gas}

One obvious observation regarding the existence of an upper mass limit for star cluster formation is that the total stellar mass formed should be significantly higher than that of the most massive cluster. This is because young star clusters of the type likely to evolve into GCs and UCDs do not generally form alone, but in fact form in large numbers during violent galaxy interactions (see e.g. the YMC populations of NGC 1316 and NGC 7252; Goudfrooij 2012; Bastian et al. 2013). From Section 4.1 we see that we expect that the most massive star clusters form in major galaxy interactions where the ISM density and turbulence is high. We also expect major galaxy mergers to be a site of formation for massive clusters because sufficient quantities of gas are available, and the star clusters can form on orbits that keep them away from the galaxy centre or disc, which protects them from total disruption through dynamical friction within a short period, allowing them to survive until the present epoch.

Observations of young star clusters in merger systems show that the number of clusters follows a power-law dependence on cluster mass of the form $\mathrm{d} N / \mathrm{d} M \propto M^{-\beta}$ with $\beta=2$ (Fall, Chandar \& Whitmore 2009). Furthermore, observations indicate that the most massive star clusters found in these nearby merger remnants are consistent with the expectations of simply statistically sampling from the same power law as the bulk cluster population (see e.g. Schweizer \& Seitzer 2007, Goudfrooij et al. 2004, Whitmore et al. 2002, 2010, and Miller et al. 1997, for the cases of NGC 34, NGC 1316, NGC 3610, NGC 4038/39, and NGC 7252, respectively).

As Elmegreen, Malhotra \& Rhoads (2012) demonstrate, this mass dependence can be reformulated to provide a prediction for the total stellar mass formed in a given star-forming period that produces a cluster of mass $M$ (their equation 2 )

$$
\begin{aligned}
M_{\text {total }}= & \eta_{\mathrm{c}}^{-1} M+\eta_{\mathrm{c}}^{-1}(\beta-1) M^{\beta-1} \\
& \times\left(\frac{M^{2-\beta}-M_{\min }^{2-\beta}}{2-\beta}, \ln \left[\frac{M}{M_{\min }}\right]\right) .
\end{aligned}
$$

Here $\eta_{\mathrm{c}}$ is the fraction of stars formed in star clusters. Following Elmegreen et al. (2012) we assume a conservative fraction of 0.25 , as the value of $\eta_{\mathrm{c}}$ has been claimed to vary significantly with local physical conditions (Silva-Villa, Adamo \& Bastian 2013). Adamo $\&$ Bastian (2015) suggest that the fraction of stars forming in bound clusters varies from $\sim 3$ per cent in quiescent dwarf galaxies, to $\sim 50$ per cent or more in the most intense starbusts. Kruijssen 2012 likewise suggest a range of between $\sim 3$ per cent in the lowest density galaxies and 70 per cent in the highest density systems. Finally, the E-MOSAICS simulations (Pfeffer et al. 2018) indicate cluster formation efficiencies that are generally 20-30 per cent at $z$ $=6$, can reach $\sim 80$ per cent during bursts at intermediate redshifts, and decline to $\sim 1$ per cent at $z=0$ for their simulated Milky Way analogues.

We note however, that recently Chandar et al. (2017) claim that the apparent variation in the fraction of stars forming in clusters with environmental conditions is in fact due to observational inconsistencies, and in particular the fact that the times since cluster formation is different in each case, leading to differing amounts of cluster dissolution.

Nevertheless the value for the fraction of stars formed in clusters that they determine ( $24 \pm 9$ per cent) is consistent with our adopted value. $M_{\min }$ is the minimum bound cluster mass, which in line with Elmegreen et al. (2012) is assumed to be $10 \mathrm{M}_{\odot}$ here.

Note that in using this derivation we explicitly assume that there is no physical truncation of the initial cluster mass function, clusters 
can form up to any mass, as long as sufficient gas is available. This is in contrast to a cluster mass function of the form typically found for disc galaxies, where an exponential truncation (Schechter 1976), generally occurs at a few $\times 10^{5} \mathrm{M}_{\odot}$ (Gieles et al. 2006; Bastian 2008; Gieles 2009; Larsen 2009; Kruijssen 2014; Adamo et al. 2015, 2017).

Using this equation we find that in order to form a most massive cluster with mass $M=10^{8} \mathrm{M}_{\odot}$ the total mass of stars formed in the star formation event is $7 \times 10^{9} \mathrm{M}_{\odot}$. We note that these values are consistent with those found by the E-MOSAICS simulations (see fig. 5 of Pfeffer et al. 2018). They are also broadly consistent with the findings of Ma et al. (2019), who find that to form a cluster of mass $M_{\mathrm{cl}}$ requires the formation of $20 M_{\mathrm{cl}}$ of stars in the galaxy as a whole. As the total efficiency of the conversion of molecular gas to stars $\left(\eta_{\star}\right)$ is never unity, the total amount of molecular gas required will be significantly larger. Assuming the average star formation efficiency is similar to that observed in Milky Way molecular clouds (i.e. 2 per cent; Leisawitz, Bash \& Thaddeus 1989), the total molecular gas required would be of the order $4 \times 10^{11} \mathrm{M}_{\odot}$. Alternatively, the required molecular gas mass could be reduced by a factor of 10-20 if the star formation efficiencies were assumed to be in the range thought to be required for a star cluster to remain bound after gas expulsion (i.e. SFE > 20-40 per cent; Parmentier et al. 2008; Smith et al. 2011). Such high average star formation efficiencies are observationally motivated, as observations of starbursts indicate that they are forming stars more efficiently than local spiral discs by factors of $>10$ (see e.g. Meier et al. 2010; Silverman et al. 2015).

Therefore, we arrive at a required molecular gas mass in the range from $\sim 1 \times 10^{10}$ to $4 \times 10^{11} \mathrm{M}_{\odot}$ in order to form a most massive star cluster with stellar mass of $10^{8} \mathrm{M}_{\odot}$ at birth. Note that this molecular gas mass does not necessarily all have to be concentrated in a single star-forming complex, but at least $10^{8} \times \eta_{\star}^{-1} \mathrm{M}_{\odot}$ must be located within a single bound structure to create the most massive cluster.

The need for such enormous quantities of cold molecular gas naturally places a strong constraint on the probability of forming such massive clusters. Even massive disc galaxies, such as the Milky Way or M51, typically have total molecular gas masses of only around $5 \times 10^{9}$ (Shetty et al. 2007; Schinnerer et al. 2013) and no single cloud has a mass that exceeds $2 \times 10^{7} \mathrm{M}_{\odot}$ (Colombo et al. 2014). In fact, in a study cross-matching ALFALFA and SDSS data of $>11000$ galaxies out to $z=0.06$ Maddox et al. (2015) find few galaxies with cold gas mass $>10^{10} \mathrm{M}_{\odot}$ and none with $>10^{11} \mathrm{M}_{\odot}$. In contrast, at $z>1.5$ cold gas masses of $>10^{11} \mathrm{M}_{\odot}$ are seen, at least for the most massive galaxies thought to be likely progenitors of early-type galaxies (see e.g. Tacconi et al. 2013; Scoville et al. 2016; Rudnick et al. 2017). However, no galaxies with gas mass $>10^{12} \mathrm{M}_{\odot}$ are seen at $z>2$ (or anywhere else), despite being easier to detect. It is therefore plausible that the lack of star clusters with mass $>10^{8} \mathrm{M}_{\odot}$ could be down to the fact that there simply are not any galaxies/mergers where sufficient cold gas is available at any one time to create them.

This scenario is therefore statistical in nature; there need not be a physical limitation of gas physics which prevents larger clusters forming, it is simply that our Universe rarely, if ever, brings together enough cold gas to create such clusters, leading to a practical limit on the maximum cluster mass found in a reasonable volume. It might therefore be speculated that the upper limit produced by this scenario is the result of cosmology, with the interplay of the initial matter power spectrum and the expansion rate of the Universe ultimately setting how much gas can be accumulated at any one epoch.

\subsection{Scenario C: shear}

Reina-Campos \& Kruijssen (2017) present a simple analytical model to determine the maximum mass of star clusters. They suggest that the limiting mass is set by a combination of stellar feedback and environmental shear. Their results indicate that cluster formation within Milky Way-like spiral discs will typically be feedback limited beyond $4 \mathrm{kpc}$, while more massive higher redshift star formation will likely be shear limited at all radii, a change driven by the large increase in gas surface density at higher redshift. Their model predicts maximum star cluster masses that broadly agree with our limit at high redshift $\left(\sim 10^{8}-10^{9} \mathrm{M}_{\odot}\right)$, with a reduction to $\sim 10^{4}-10^{5} \mathrm{M}_{\odot}$ for local galaxy discs. However, this model assumes cluster formation occurs within a differentially rotating disc in hydrostatic equilibrium, an assumption that has been shown to be valid for high-redshift galaxies because despite the clumpy and chaotic nature of young galaxies, simulations indicate that star formation is still restricted to relatively thin discs (Meng, Gnedin \& Li 2019). However, it remains unclear how reliable an assumption this would be for the progenitors of today's GCs and UCDs, as these objects are now found on orbits that keep them well away from the densest regions of their host galaxies, where dynamical friction would rapidly lead to their destruction. Presumably an interaction would be required to eject the protostar clusters on to orbits with longer dynamical friction time-scales, it is not currently clear whether such interactions would be common enough to explain the observed abundance of massive star clusters. Nevertheless, this model if extended to describe the shear environment of a major merger or starburst holds promise for explaining the maximum star cluster mass across all mass scales.

\subsection{Scenario D: stellar feedback}

Stellar feedback alone may be able to explain the maximum star cluster mass, subject to some uncertainty regarding star formation efficiencies. Massive young stars emit copious amounts of highenergy photons that deposit momentum into the surrounding ISM, when this exceeds the force of gravity the gas is expelled and any further star formation is curtailed. As discussed in, e.g. Murray, Quataert \& Thompson (2010), Hopkins et al. (2010), Rahner et al. (2017), Crocker et al. (2018), and Grudić et al. (2019) this behaviour is analogous to the Eddington limit for stars. However, one important difference is that the ISM of star-forming regions is dusty, and the opacity of dusty gas is much higher than the electron scattering opacity found in stars. Therefore radiation from young massive star clusters could efficiently act to restrict their own growth.

When examining a range of dense stellar systems from GCs to galaxy spheroids, Hopkins et al. (2010) find a nearly constant maximum central stellar surface mass density. They attribute this maximum surface mass density to stellar feedback reaching an Eddington-like limit that regulates the growth of dense star-forming regions. They also show that for certain assumptions this Eddingtonlike limit is reached for a gas surface mass density of $\Sigma_{\text {gas }}=10^{11}$ $10^{12} \mathrm{M}_{\odot} \mathrm{kpc}^{-2}$. Recently Crocker et al. (2018) confirm this result as being consistent with that expected to be caused by direct and indirect radiation pressure from the young stellar cluster. Converting this apparent limit into the correct area for typical massive UCDs (which have $R_{e} \sim 20-100 \mathrm{pc}$ ) and assuming the efficiency of gas to stellar mass conversion described in Section 4.2 (i.e. 2-40 per cent) does in fact produce stellar masses in the correct range for the most massive UCDs $\left(>10^{7} \mathrm{M}_{\odot}\right)$. 
More detailed simulation work is required to constrain the expected range of star cluster formation efficiencies for this scenario (efficiencies as high as 90 per cent are found in the radiation limited case by Crocker et al. 2018), and to include other sources of energy injection (e.g. prompt $\mathrm{SN}$ ) to see how these will impact the final bound cluster mass. It may also prove the case that even when this effect operates the maximum cluster mass is still limited by one of the other scenarios outlined, for example by limitations on the availability of sufficient gas. Recently Grudić et al. (2019) have taken steps in exactly this direction to produce a modified model in which the upper limit of the stellar surface density is caused by stellar feedback becoming ineffective above some critical threshold, thereby causing the supply of gas to be rapidly expended before the system can contract to higher density.

\section{DISCUSSION}

All four proposed mechanisms currently provide plausible explanations for why star clusters would experience a maximum mass limit. Additional observational and simulation work will be required to determine which (if any) is responsible for the observed upper mass limit.

The first two mechanisms are essentially statistical in nature. This is a strength as it means that they can potentially naturally explain not just the existence of the upper mass limit, but also the distribution of masses of star clusters. For scenario A a distribution of pressures and densities throughout the merger leads to a range of initial star cluster sizes and masses. For scenario B a lower total galactic gas mass populates less far up the star cluster mass function, but still forms clusters up to that mass, and furthermore could produce the right mass function for star clusters, assuming the correct GMC mass function and differential survival of YMCs to become GCs based on their mass.

This statistical nature is also a problem as in order to determine their efficacy in producing the observed limit the observational or simulated data must be more comprehensive. For example, to accurately test mechanism A (extreme ISM conditions) will require simulating many different galaxy mergers and starbursts with the resolution (ideally more) used by Renaud et al. (2014, 2015), followed by comparing the produced mass distributions of surviving star clusters with those observed in massive early-type galaxies. Likewise, for mechanism B (insufficient gas supply) it is necessary to examine the molecular gas reservoirs and star formation activity of a large ensemble of simulated galaxies, and to examine how the mass function of surviving star clusters correlates with the available gas reservoirs at the epoch when they formed.

Mechanism C (shear) is potentially similar, in that the correct distribution of stellar feedback and shear could lead to both the observed mass function of star clusters, and their ultimate upper mass limit. Simulations similar to those required to investigate mechanism A, plus observations of interacting and quiescent galaxies will eventually demonstrate whether the range of stellar feedback and shear environments present in such galaxies matches those required to explain the full mass range of star clusters. In the near future the ongoing simulations of the E-MOSAICS project (Pfeffer et al. 2018) which incorporate the formation and evolution of star cluster populations following the prescriptions of Kruijssen (2012) and Reina-Campos \& Kruijssen (2017) into the EAGLE simulations of galaxy formation (Crain et al. 2015; Schaye et al. 2015) should demonstrate the efficacy of this scenario.

Further work is necessary to demonstrate if mechanism D (stellar feedback) can produce not only a maximum upper mass limit, but also explain the observed mass function of star clusters, either alone or in combination with one of the other scenarios.

Finally it is worth noting that the mechanisms described here apply only to the genuine star cluster population of UCDs. Those UCDs formed by the liberation of galaxy nuclei during tidal interactions would not be expected to be limited by any of the processes outlined, principally because galaxy nuclei can undergo repeated bursts of star formation (see e.g. Norris et al. 2015). This ensures the expected mass function of former nuclei should extend to significantly higher mass than that of the genuine star cluster population, and the presence of any upper mass limit to such objects becomes difficult to discern due to overlap with other similar objects like compact ellipticals.

\section{CONCLUSIONS}

We have assembled the most comprehensive sample of CSS yet currently robustly classified. This sample is the largest available compilation in the intermediate luminosity/mass regime, where the division between star clusters and galaxies is most uncertain.

Using this catalogue we have strengthened the existing evidence for the existence of an upper initial mass limit of surviving genuine star clusters at birth of around $10^{8} \mathrm{M}_{\odot}$. Definitively demonstrating the exact location of this limit will require future volume-limited, spectroscopically confirmed and highly complete CSS surveys.

We have examined four possible mechanisms responsible for the lack of bona fide star clusters with stellar masses $>10^{8} \mathrm{M}_{\odot}$, and conclude that all are plausible. Further simulation work looking at the ensemble properties of the galaxy population at higher redshift (to examine the cold gas distributions), additional higher resolution simulations of major mergers (to check the distributions of most massive clusters produced), and focused simulations of single massive star cluster formation (to examine the effect of stellar feedback) will be required to determine which is the principal effect.

\section{ACKNOWLEDGEMENTS}

The authors would like to thank the anonymous referee along with Sharon Meidt and Brent Groves for their extremely helpful comments that greatly improved this paper.

GvdV acknowledges funding from the European Research Council (ERC) under the European Union's Horizon 2020 research and innovation programme under grant agreement no. 724857 (Consolidator Grant ArcheoDyn).

\section{REFERENCES}

Adamo A., Bastian N., 2015, preprint (arXiv:1511.08212)

Adamo A., Kruijssen J. M. D., Bastian N., Silva-Villa E., Ryon J., 2015, MNRAS, 452, 246

Adamo A. et al., 2017, ApJ, 841, 131

Afanasiev A. V. et al., 2018, MNRAS, 477, 4856

Ahn C. P. et al., 2017, ApJ, 839, 72

Ahn C. P. et al., 2018, ApJ, 858, 102

Bastian N., 2008, MNRAS, 390, 759

Bastian N., Schweizer F., Goudfrooij P., Larsen S. S., Kissler-Patig M., 2013, MNRAS, 431, 1252

Bekki K., Couch W. J., 2003, ApJ, 596, L13

Bournaud F., Duc P.-A., Emsellem E., 2008, MNRAS, 389, L8

Bouwens R. J., van Dokkum P. G., Illingworth G. D., Oesch P. A., Maseda M., Ribeiro B., Stefanon M., Lam D., 2017, preprint (arXiv:1711.02090)

Brodie J. P., Romanowsky A. J., Strader J., Forbes D. A., 2011, AJ, 142, 199 
Cabrera-Ziri I., Bastian N., Davies B., Magris G., Bruzual G., Schweizer F., 2014, MNRAS, 441, 2754

Cabrera-Ziri I. et al., 2015, MNRAS, 448, 2224

Caso J. P., Bassino L. P., Richtler T., Smith Castelli A. V., Faifer F. R., 2013, MNRAS, 430, 1088

Caso J. P., Bassino L. P., Richtler T., Calderón J. P., Smith Castelli A. V., 2014, MNRAS, 442, 891

Chandar R., Fall S. M., Whitmore B. C., Mulia A. J., 2017, ApJ, 849, 128

Chiboucas K. et al., 2011, ApJ, 737, 86

Choksi N., Gnedin O. Y., 2019, preprint (arXiv:1905.05199)

Choksi N., Gnedin O. Y., Li H., 2018, MNRAS, 480, 2343

Clauwens B., Schaye J., Franx M., Bower R. G., 2018, MNRAS, 478, 3994

Colombo D. et al., 2014, ApJ, 784, 3

Côté P. et al., 2004, ApJS, 153, 223

Crain R. A. et al., 2015, MNRAS, 450, 1937

Crocker R. M., Krumholz M. R., Thompson T. A., Baumgardt H., Mackey D., 2018, MNRAS, 481, 4895

Da Rocha C., Mieske S., Georgiev I. Y., Hilker M., Ziegler B. L., Mendes de Oliveira C., 2011, A\&A, 525, A86

Drinkwater M. J., Jones J. B., Gregg M. D., Phillipps S., 2000, Publ. Astron. Soc. Aust., 17, 227

Du M. et al., 2019, ApJ, 875, 58

Elmegreen B. G., 2018, ApJ, 869, 119

Elmegreen B. G., Malhotra S., Rhoads J., 2012, ApJ, 757, 9

Faifer F. R. et al., 2011, MNRAS, 416, 155

Faifer F. R., Escudero C. G., Scalia M. C., Smith Castelli A. V., Norris M., De Rossi M. E., Forte J. C., Cellone S. A., 2017, A\&A, 599, L8

Falgarone E. et al., 2017, Nature, 548, 430

Fall S. M., Chandar R., Whitmore B. C., 2009, ApJ, 704, 453

Fellhauer M., Kroupa P., 2002, MNRAS, 330, 642

Ferrarese L. et al., 2012, ApJS, 200, 4

Forbes D. A., Bridges T., 2010, MNRAS, 404, 1203

Forbes D. A., Kroupa P., 2011, Publ. Astron. Soc. Aust., 28, 77

Forbes D. A., Norris M. A., Strader J., Romanowsky A. J., Pota V., Kannappan S. J., Brodie J. P., Huxor A., 2014, MNRAS, 444, 2993

Galleti S., Federici L., Bellazzini M., Fusi Pecci F., Macrina S., 2004, A\&A, 416, 917

Georgiev I. Y., Böker T., 2014, MNRAS, 441, 3570

Gieles M., 2009, MNRAS, 394, 2113

Gieles M., Larsen S. S., Scheepmaker R. A., Bastian N., Haas M. R., Lamers H. J. G. L. M., 2006, A\&A, 446, L9

Goodman M., Bekki K., 2018, MNRAS, 478, 3564

Goudfrooij P., 2012, ApJ, 750, 140

Goudfrooij P., Gilmore D., Whitmore B. C., Schweizer F., 2004, ApJ, 613, L121

Gratton R. G., Carretta E., Bragaglia A., 2012, A\&AR, 20, 50

Grudić M. Y., Hopkins P. F., Quataert E., Murray N., 2019, MNRAS, 483, 5548

Harris W. E., 1996, AJ, 112, 1487

Harris W. E. et al., 2014, ApJ, 797, 128

Haşegan M. et al., 2005, ApJ, 627, 203

Hendricks B., Koch A., Lanfranchi G. A., Boeche C., Walker M., Johnson C. I., Peñarrubia J., Gilmore G., 2014, ApJ, 785, 102

Hilker M., 2006, preprint (arXiv:e-print)

Hilker M., 2009, preprint (arXiv:0906.0776)

Hilker M., Infante L., Vieira G., Kissler-Patig M., Richtler T., 1999, A\&AS, 134,75

Hopkins P. F., Murray N., Quataert E., Thompson T. A., 2010, MNRAS, 401, L19

Huxor A. P., Phillipps S., Price J., Harniman R., 2011, MNRAS, 414, 3557

Into T., Portinari L., 2013, MNRAS, 430, 2715

Janz J. et al., 2016, MNRAS, 456, 617

Jennings Z. G. et al., 2015, ApJ, 812, L10

Jordán A. et al., 2007, ApJS, 171, 101

Kim J.-h. et al., 2018, MNRAS, 474, 4232

Kissler-Patig M., Jordán A., Bastian N., 2006, A\&A, 448, 1031

Koch A., Grebel E. K., Wyse R. F. G., Kleyna J. T., Wilkinson M. I., Harbeck D. R., Gilmore G. F., Evans N. W., 2006, AJ, 131, 895
Kormendy J., Fisher D. B., Cornell M. E., Bender R., 2009, ApJS, 182, 216 Kroupa P., 2001, MNRAS, 322, 231

Kruijssen J. M. D., 2012, MNRAS, 426, 3008

Kruijssen J. M. D., 2014, Class. Quantum Gravity, 31, 244006

Larsen S. S., 2009, A\&A, 494, 539

Leaman R., VandenBerg D. A., Mendel J. T., 2013, MNRAS, 436, 122

Leisawitz D., Bash F. N., Thaddeus P., 1989, ApJS, 70, 731

Li Y., Mac Low M.-M., Klessen R. S., 2004, ApJ, 614, L29

Li H., Gnedin O. Y., Gnedin N. Y., Meng X., Semenov V. A., Kravtsov A. V., 2017, ApJ, 834, 69

Liu C. et al., 2015, ApJ, 812, 34

Ma X. et al., 2019, preprint (arXiv:1906.11261)

Maddox N., Hess K. M., Obreschkow D., Jarvis M. J., Blyth S.-L., 2015, MNRAS, 447, 1610

Madrid J. P., Donzelli C. J., 2013, ApJ, 770, 158

Maji M., Zhu Q., Li Y., Charlton J., Hernquist L., Knebe A., 2017, ApJ, 844,108

Maraston C., Bastian N., Saglia R. P., Kissler-Patig M., Schweizer F., Goudfrooij P., 2004, A\&A, 416, 467

Matsui H. et al., 2012, ApJ, 746, 26

Meier D. S., Turner J. L., Beck S. C., Gorjian V., Tsai C.-W., Van Dyk S. D., 2010, AJ, 140, 1294

Meng X., Gnedin O. Y., Li H., 2019, MNRAS, 486, 1574

Mieske S., Hilker M., Infante L., 2004, A\&A, 418, 445

Mieske S., Hilker M., Misgeld I., 2012, A\&A, 537, A3

Miller B. W., Whitmore B. C., Schweizer F., Fall S. M., 1997, AJ, 114, 2381

Minniti D., Kissler-Patig M., Goudfrooij P., Meylan G., 1998, AJ, 115, 121

Misgeld I., Hilker M., 2011, MNRAS, 414, 3699

Misgeld I., Mieske S., Hilker M., Richtler T., Georgiev I. Y., Schuberth Y., 2011, A\&A, 531, A4

Murray N., Quataert E., Thompson T. A., 2010, ApJ, 709, 191

Norris M. A., Kannappan S. J., 2011, MNRAS, 414, 739

Norris M. A. et al., 2014, MNRAS, 443, 1151

Norris M. A., Escudero C. G., Faifer F. R., Kannappan S. J., Forte J. C., van den Bosch R. C. E., 2015, MNRAS, 451, 3615

Oser L., Ostriker J. P., Naab T., Johansson P. H., Burkert A., 2010, ApJ, 725, 2312

Parmentier G., Goodwin S. P., Kroupa P., Baumgardt H., 2008, ApJ, 678, 347

Penny S. J., Forbes D. A., Conselice C. J., 2012, MNRAS, 422, 885

Penny S. J., Forbes D. A., Strader J., Usher C., Brodie J. P., Romanowsky A. J., 2014, MNRAS, 439, 3808

Pfeffer J., Baumgardt H., 2013, MNRAS, 433, 1997

Pfeffer J., Griffen B. F., Baumgardt H., Hilker M., 2014, MNRAS, 444, 3670

Pfeffer J., Hilker M., Baumgardt H., Griffen B. F., 2016, MNRAS, 458, 2492

Pfeffer J., Kruijssen J. M. D., Crain R. A., Bastian N., 2018, MNRAS, 475, 4309

Phillipps S., Drinkwater M. J., Gregg M. D., Jones J. B., 2001, ApJ, 560, 201

Qu Y. et al., 2017, MNRAS, 464, 1659

Rahner D., Pellegrini E. W., Glover S. C. O., Klessen R. S., 2017, MNRAS, 470,4453

Reina-Campos M., Kruijssen J. M. D., 2017, MNRAS, 469, 1282

Renaud F. et al., 2013, MNRAS, 436, 1836

Renaud F., Bournaud F., Kraljic K., Duc P.-A., 2014, MNRAS, 442, L33

Renaud F., Bournaud F., Duc P.-A., 2015, MNRAS, 446, 2038

Rodriguez-Gomez V. et al., 2016, MNRAS, 458, 2371

Rudnick G. et al., 2017, ApJ, 849, 27

Schaye J. et al., 2015, MNRAS, 446, 521

Schechter P., 1976, ApJ, 203, 297

Schinnerer E. et al., 2013, ApJ, 779, 42

Schweizer F., Seitzer P., 2007, AJ, 133, 2132

Schweizer F., Seitzer P., Whitmore B. C., Kelson D. D., Villanueva E. V., 2018, ApJ, 853, 54

Scoville N. et al., 2016, ApJ, 820, 83

Seth A. C. et al., 2014, Nature, 513, 398 
Shetty R., Vogel S. N., Ostriker E. C., Teuben P. J., 2007, ApJ, 665, 1138

Silva-Villa E., Adamo A., Bastian N., 2013, MNRAS, 436, L69

Silverman J. D. et al., 2015, ApJ, 812, L23

Smith R., Fellhauer M., Goodwin S., Assmann P., 2011, MNRAS, 414, 3036

Starkenburg E. et al., 2013, A\&A, 549, A88

Strader J., Brodie J. P., Spitler L., Beasley M. A., 2006, AJ, 132, 2333

Swinbank A. M. et al., 2011, ApJ, 742, 11

Tacconi L. J. et al., 2013, ApJ, 768, 74

Taylor M. A., Puzia T. H., Harris G. L., Harris W. E., Kissler-Patig M., Hilker M., 2010, ApJ, 712, 1191

Usher C., Pfeffer J., Bastian N., Kruijssen J. M. D., Crain R. A., ReinaCampos M., 2018, MNRAS, 480, 3279 van der Wel A. et al., 2014, ApJ, 788, 28

Vanzella E. et al., 2017, MNRAS, 467, 4304

Voggel K., Hilker M., Richtler T., 2016, A\&A, 586, A102

Whitmore B. C., Schweizer F., Kundu A., Miller B. W., 2002, AJ, 124, 147

Whitmore B. C. et al., 2010, AJ, 140, 75

Willman B., Strader J., 2012, AJ, 144, 76

Zhang H.-X. et al., 2015, ApJ, 802, 30

This paper has been typeset from a $\mathrm{TE}_{\mathrm{E}} \mathrm{X} / \mathrm{A} \mathrm{T}_{\mathrm{E}} \mathrm{X}$ file prepared by the author. 